\title{
DESCRIÇÃO DE UMA NOVA ESPÉCIE DE RHOPALURUS THORELL, 1876 (SCORPIONES: BUTHIDAE) DO NORDESTE BRASILEIRO
}

\author{
Ângelo Ricardo I. P. Lenarducci' ${ }^{1}$ Ricardo Pinto-da-Rocha ${ }^{2}$ \& Sylvia Marlene Lucas ${ }^{1}$
}

Biota Neotropica v5 (n1a)-http://www.biotaneotropica.org.br/v5n1a/pt/abstract?taxonomic-review+bn017051a2005

Recebido em 07/12/2003

Publicado em 01/02/2005

\begin{abstract}
${ }^{1}$ Instituto Butantan, Laboratório de Artrópodes Peçonhentos, Av. Vital Brasil, 1500, São Paulo, CEP: 05530-900, Brasil, e-mail: angelolenarducci@ig.com.br, sylvialucas@,butantan.gov.br.

${ }^{2}$ Departamento de Zoologia, Instituto de Biociências, Universidade de São Paulo, Caixa Postal 11461, CEP: 05422-970, São Paulo, SP, Brasil, e-mail: ricrocha@usp.br.
\end{abstract}

\begin{abstract}
Rhopalurus guanambiensis n. sp. is described from Guanambi, state of Bahia, northeastern Brazil. The new species is related to R. lacrau Lourenço \& Pinto-da-Rocha, 1997 (Itaeté, state of Bahia) by having the same number of oblique rows of granules on the movable finger of the pedipalpal chela, same number of keels on metasomal segments I, III, IV, V and similar body size; it is related to R. crassicauda Di Caporiacco, 1947 (state of Roraima) by having the same number of keels on metasomal segments I, II, IV and V, last two metasomal segments darker colored and enlarged, and similar body size. It is distinguished from $R$. lacrau by the different color of the carapace, meso and metasomal segments IV-V, higher number of pectinal teeth and metasomal segment II with ten carinae; and from $R$. crassicauda by the lower number of oblique rows of granules on the movable finger of the pedipalp chela, metasomal segment III with eight carinae; segment V with vestigial keels and by the telson with a strong subaculear tubercle. The males of R. guanambiensis are distinguished from the female by having more robust pedipalp chelae and by the enlarged metasomal segments IV and V. This species raises the total number of species of the genus Rhopalurus in Brazil to nine.
\end{abstract}

Key words: Scorpiones, Buthidae, Rhopalurus guanambiensis n. sp., Northeastern Brazil.

\section{Resumo}

Rhopalurus guanambiensis n.sp. é descrita de Guanambi, Estado da Bahia, nordeste do Brasil. A nova espécie aproxima-se de R. lacrau Lourenço \& Pinto-da-Rocha, 1997 (Itaeté, Estado da Bahia) por apresentar o mesmo número de séries de grânulos no dedo móvel da quela do pedipalpo, igual quantidade de carenas nos segmentos metasomais I, III, IV e V e pelo tamanho do corpo semelhante; aproxima-se de $R$. crassicauda Di Caporiacco, 1947 (Estado da Roraima) por apresentar o mesmo número de carenas nos segmentos metasomais I, II, IV e V, pela coloração mais escura dos dois últimos segmentos metasomais, que são dilatados e por apresentar aproximadamente o mesmo tamanho. Distingue-se de $R$. lacrau pela coloração diferente da carapaça, mesosoma e dos segmentos metasomais IV-V, maior número de dentes pectínios e pela presença de dez carenas no segmento metasomal II; de R. crassicauda, devido ao menor número de séries de grânulos no dedo móvel da quela do pedipalpo, maior número de dentes pectíneos, presença de oito carenas no segmento metasomal III e por apresentar um tubérculo subaculear acentuado no telson e carenas do segmento $\mathrm{V}$ do metasoma vestigiais. $\mathrm{O}$ macho de R. guanambiensis distingue-se da fêmea pela mão mais robusta e pela dilatação dos dois últimos segmentos do metasoma. A descrição desta espécie amplia para nove o número de espécies que ocorrem no Brasil.

Palavras-chave: Scorpiones, Buthidae, Rhopalurus guanambiensis sp. n., Nordeste, Brasil. 


\section{Introdução}

Os escorpiões do gênero Rhopalurus Thorell, 1876 (Scorpiones, Buthidae), caracterizam-se pela presença de um aparelho estridulatório composto de duas áreas granulosas situadas no esternito III e uma região estriada nos dentes dos pentes. Movimentos rápidos dos pentes, esfregando-os sobre esta região granulosa causam a emissão de um som muitas vezes audível ao ouvido humano (Pocock 1904, Lourenço \& Cloudsley-Thompson 1995). Outros gêneros emitem som através da coxa dos pedipalpos e perna I; entre a quelícera e o prossoma; do aguilhão com segmentos caudais I-II e entre os tergitos abdominais III e VI (Acosta \& Maury 1990).

O gênero contém 13 espécies com ampla distribuição nas Antilhas, América Central e do Sul (Fet et al. 2000), das quais oito ocorrem no Brasil (Lourenço 2002). As espécies sul-americanas habitam exclusivamente formações vegetais de campos abertos como, savanas, caatingas e Llanos da Venezuela e Colômbia (Mello-Leitão 1945; Lourenço 1979, 1982, 1986).

Durante os trabalhos de reorganização da Coleção de Scorpiones do Instituto Butantan, atualmente com quase 10.000 exemplares, encontramos um lote, composto de quatro machos e uma fêmea, enviados ao Laboratório de Artrópodes em 1985 por um fornecedor de animais peçonhentos. Tratase de uma nova espécie do gênero cuja descrição é apresentada neste trabalho.

\section{Material \& método}

Os exemplares utilizados neste trabalho encontramse depositados na Coleção de Escorpiões do Instituto Butantan (IBSP-SC) e na Coleção do Museu de Zoologia da Universidade de São Paulo (MZSP). O espécimes foram examinados com o auxílio de estereomicroscópio Leica MZ 125, os desenhos confeccionados em câmara clara do mesmo modelo e as medidas com auxílio de ocular milimetrada. Foi feita a dissecção e desenhado o hemiespermatóforo direito do holótipo.

Para a elaboração da diagnose foram estudados: holótipo fêmea de R. lacrau:, Lapa do Bode, Itaeté, Bahia, Brasil, 7.IX.1993, E. Trajano leg. (MZSP-15175) e $R$. crassicauda: Fazenda São Marcos, Roraima, Brasil, VI/1964, Hoge, leg., 3J 7 $\delta^{2} 1$ (IBSP-SC 884), Normandia/Bonfim, Roraima, Brasil 26.VI.2002, Yassaka, et al. leg., $3 \delta 2 q$ (IBSP-SC 3252).

\section{Descrição}

Rhopalurus guanambiensis n. sp.

(Figs 1-11, Tabela I - II)

Tipos: Holótipo macho. Brasil, Estado da Bahia, Guanambi, (14 11' 15"S, 42 48'45"W) 1985, Neves, V. F. leg. (IBSP-SC 3404); parátipos: uma fêmea (IBSP-SC 3405); três machos (IBSP-SC 3406-3407), (MZSP-22590), com os mesmos dados do holótipo.

Etimologia: nome derivado da localidade-tipo, Guanambi, que em Tupi-guarani significa "beija-flor".

Diagnose: Rhopalurus guanambiensis n. sp. aproximase de R. lacrau Lourenço \& Pinto-da-Rocha, 1997 (Itaeté, Estado do Bahia) por apresentar o mesmo número de séries de grânulos no dedo móvel da quela do palpo, igual quantidade de carenas nos segmentos metassomais I, III, IV e V e pelo tamanho do corpo; e aproxima-se de $R$. crassicauda Di Caporiacco, 1947 (Fazenda São Marcos, Estado do Roraima) por apresentar o mesmo número de carenas nos segmentos metassomais I, II, IV e V, coloração mais escura dos dois últimos segmentos metassomais, dilatação dos segmentos caudais IV-V e tamanho aproximado. Distingue-se de $R$. lacrau pela coloração amarelo-castanho na carapaça e tergitos, segmentos metassomais IV-V marrom avermelhados ( $R$. lacrau basicamente amarelo, segmentos I ao IV amarelos), número de dentes pectíneos 27-27 (R. lacrau com 16-17 dentes) e presença de 10 carenas no segmento metassomal II ( $R$. lacrau com oito); de $R$. crassicauda, pelo número de grânulos em série no dedo móvel da quela do palpo (R. crassicauda com 89 séries), maior número de dentes pectíneos ( $R$. crassicauda 20-25), presença de oito carenas no segmento metassomal III (R. crassicauda com 10), tubérculo subaculear acentuado no telson ( $R$. crassicauda com tubérculo pequeno e não espinhoso), carenas do segmento $\mathrm{V}$ do metassoma vestigiais (R. crassicauda com carenas granulosas em toda sua extensão). A Tabela II apresenta uma comparação dos principais caracteres morfológicos apresentados pelas espécies que ocorrem no Brasil.

Descrição (holótipo macho, fig. 11): Medidas: vide Tabela I. Colorido geral do corpo amarelo castanho e pernas de colorido amarelo mais claro. Apêndices: quelíceras amarelas com as pontas dos dedos avermelhados; pedipalpos amarelos com dedos avermelhados e ponta mais clara e pernas amarelas com as pontas das garras vermelhas. Prossoma: amarelo-castanho, olhos medianos e laterais envolvidos por pigmentação negra. Mesossoma: amarelocastanho com uma faixa mais escura na margem posterior dos tergitos I-VI, tergito VII amarelo. Ventre amarelo. Metassoma: segmento I-III amarelos, IV-V marrom avermelhado; vesícula mais clara que os dois últimos segmentos; aguilhão, tubérculo subaculear e grânulos próximos à margem anterior com a ponta negra. Morfologia: Prossoma (Fig. 1) com grânulos grossos esparsos distribuídos entre olhos mediais, laterais e margem anterior; margem anterior com concavidade mediana com uma fila de grânulos grossos; cristas mediais oculares, mediais posteriores e laterais centrais com grânulos robustos; dois grânulos projetados na margem posterior seguindo a direção das carenas mediais posteriores e grânulos moderados no 


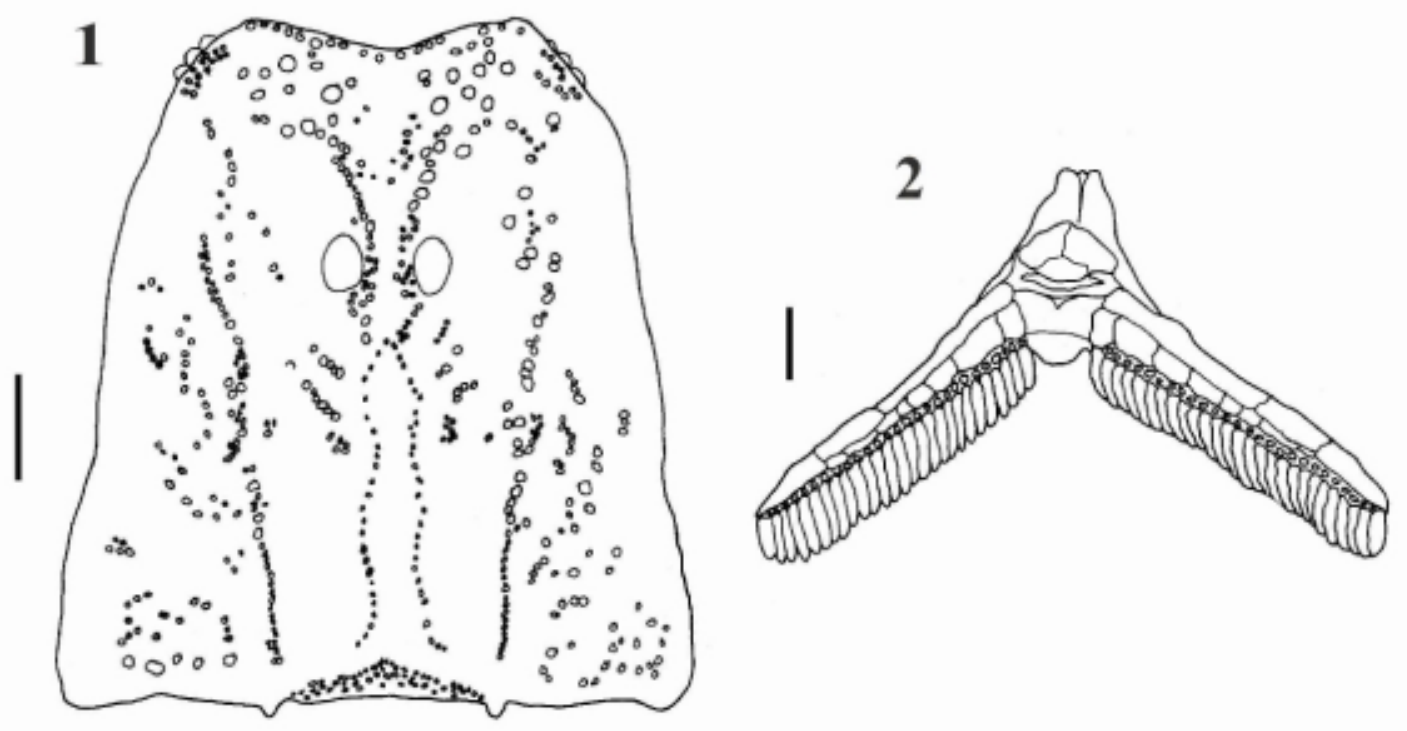

3

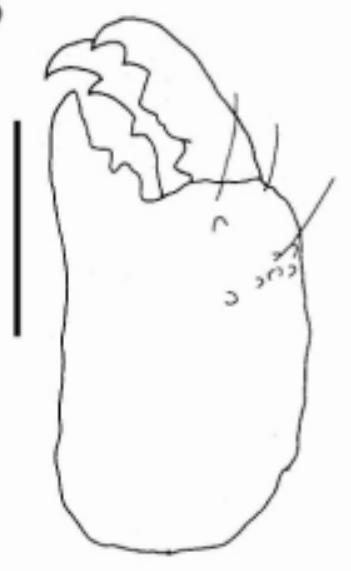

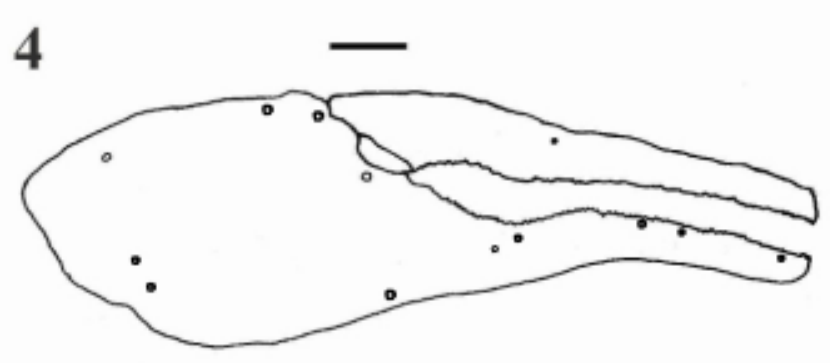

5
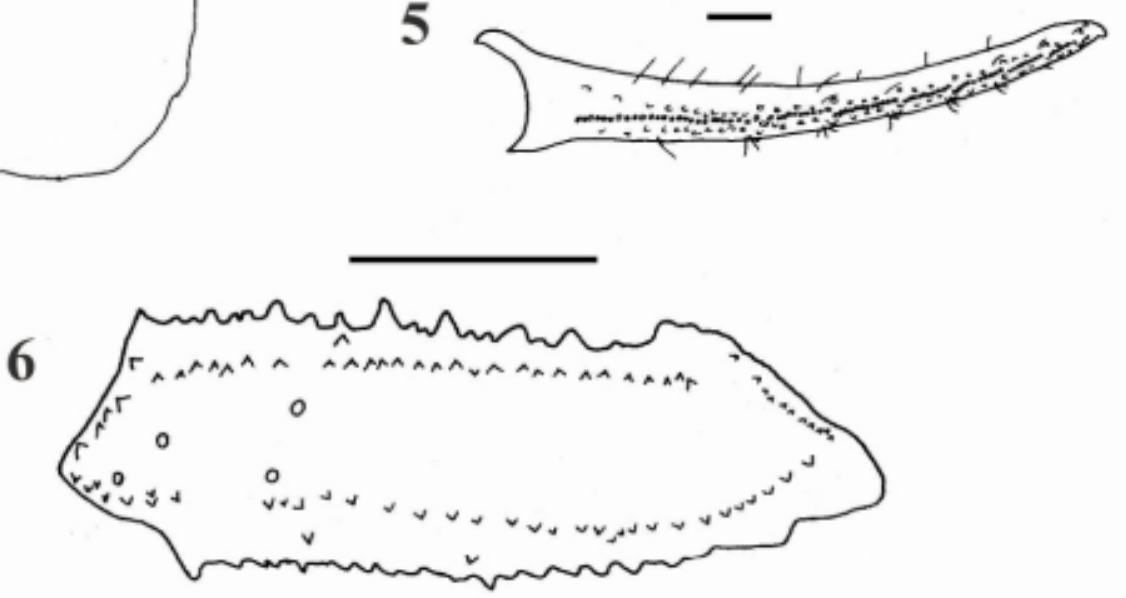

Fig. 1-6. Rhopalurus guanambiensis $n$. sp. (Escala: $1 \mathrm{~mm}$ ). 1: prossoma; 2: aspecto ventral do prossoma com opérculo genital e pentes; 3: quelícera; 4: mão com aspecto das tricobótrias; 5: disposição da granulação no dedo móvel; 6: fêmur, aspecto interno e dorsal. 


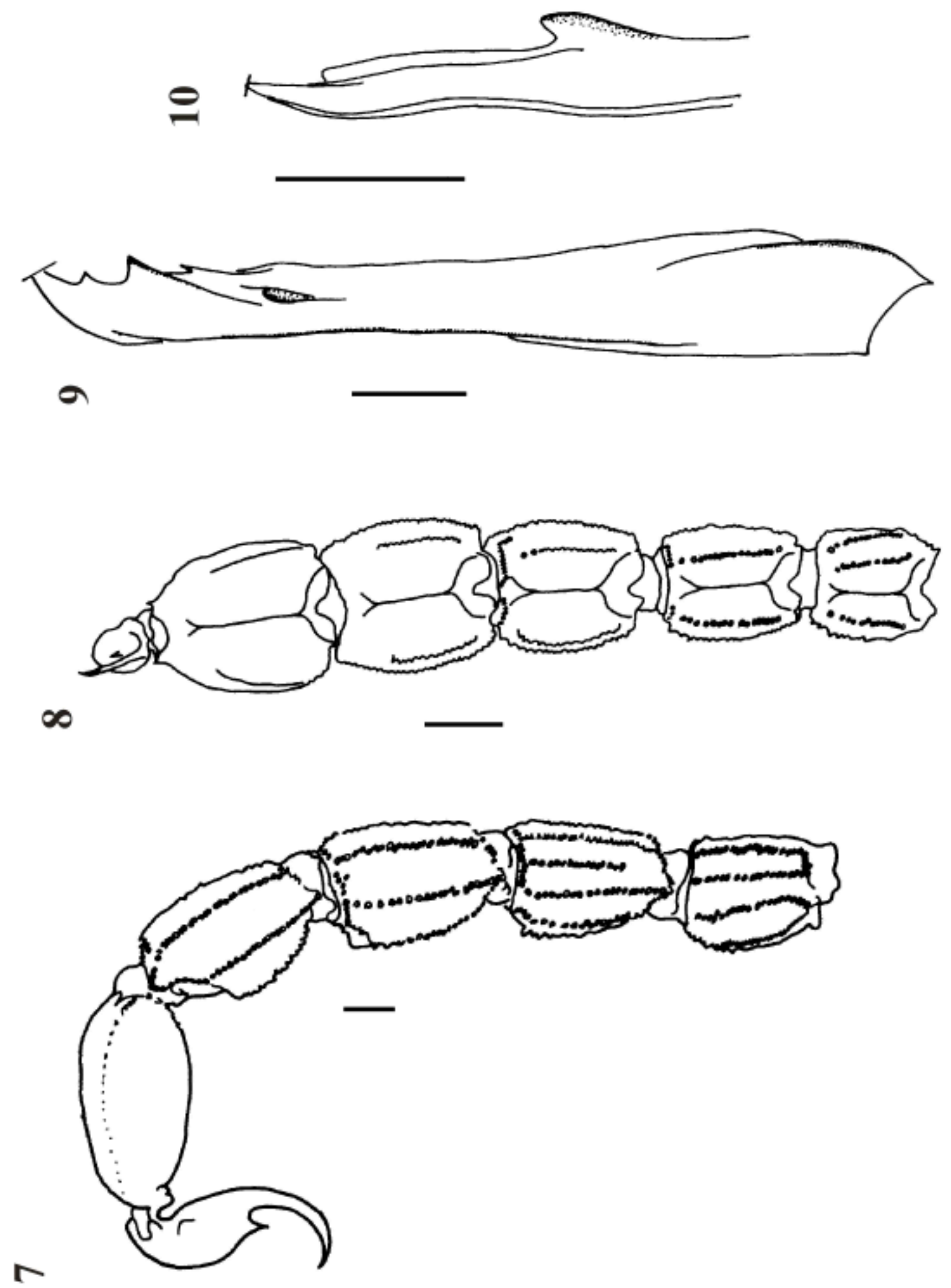




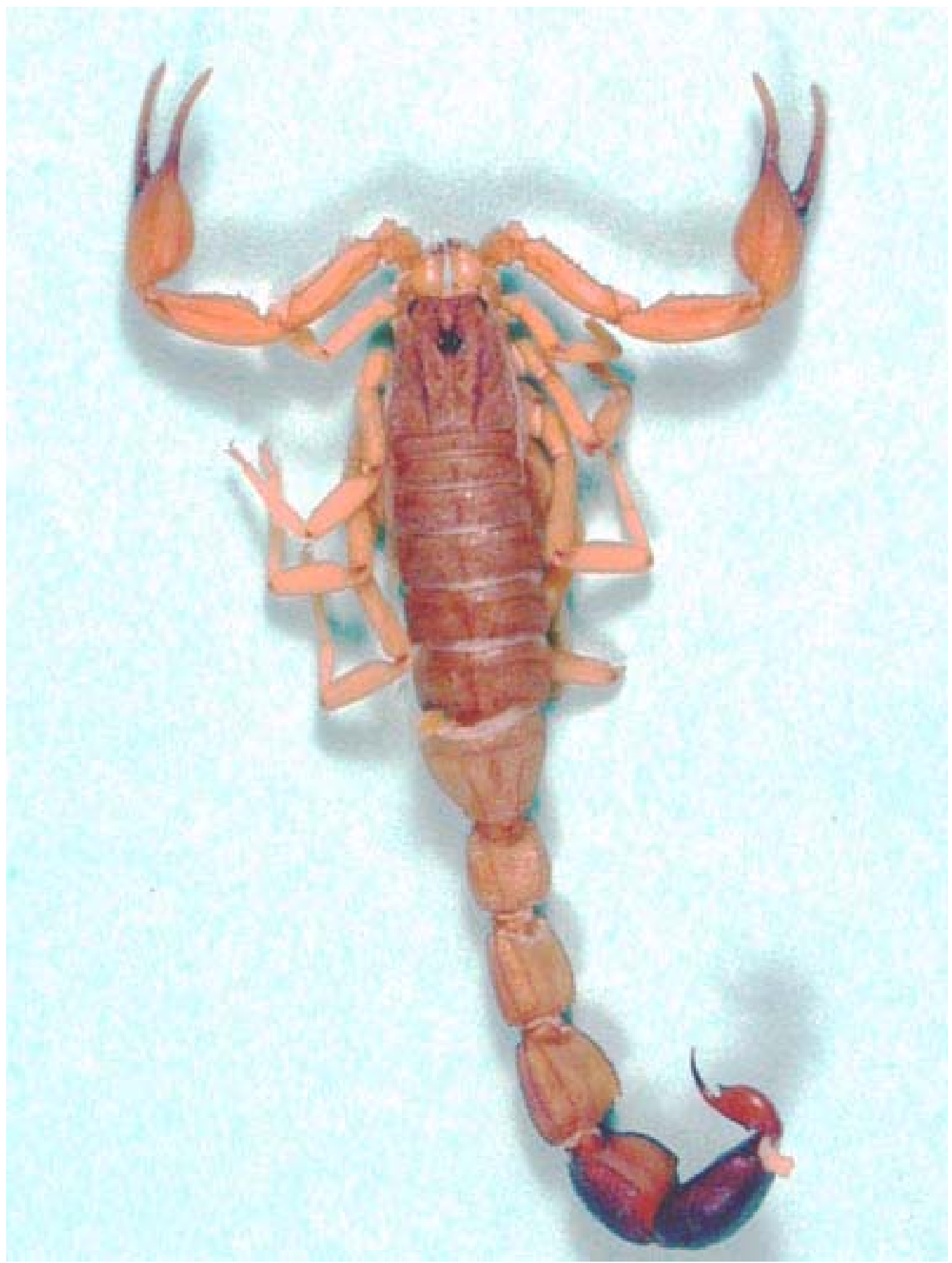

Figura 11 :Vista dorsal de: Rhopalurus guanambiensis n. sp, holótipo $\delta^{2}$ (R. Pinto-da-Rocha, 2003). 


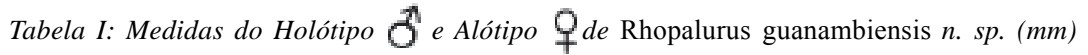

\begin{tabular}{|c|c|c|}
\hline & Holótipo $\sigma^{\Uparrow}$ & Alótipo + \\
\hline Comprimento total & 44,0 & 38,6 \\
\hline \multicolumn{3}{|l|}{ Prossoma } \\
\hline Comprimento & 4,5 & 4,5 \\
\hline Largura anterior & 2,2 & 2,0 \\
\hline Largura posterior & 4,1 & 4,5 \\
\hline \multicolumn{3}{|l|}{ Mesossoma } \\
\hline Comprimento & 12,35 & 9,5 \\
\hline \multicolumn{3}{|l|}{ Metassoma } \\
\hline Comprimento & 24,6 & 24,6 \\
\hline Segmento I comp/larg & $3,5 / 2,8$ & $3,2 / 2,9$ \\
\hline Segmento II comp/larg & $3,8 / 2,9$ & $4,0 / 3,2$ \\
\hline Segmento III comp/larg & $4,3 / 3,4$ & $4,2 / 3,5$ \\
\hline Segmento IV comp/larg & $4,5 / 3,9$ & $4,2 / 4,0$ \\
\hline Segmento V comp/larg/alt & $5,2 / 3,9 / 2,4$ & $5,4 / 3,6 / 2,3$ \\
\hline \multicolumn{3}{|l|}{ Telson } \\
\hline Comprimento & 4,2 & 4,0 \\
\hline \multicolumn{3}{|l|}{ Vesícula } \\
\hline Comprimento & 2,7 & 2,5 \\
\hline Largura & 1,1 & 1,1 \\
\hline Altura & 1,6 & 1,5 \\
\hline \multicolumn{3}{|l|}{ Aguilhão } \\
\hline Comprimento & 1,3 & 1,5 \\
\hline \multicolumn{3}{|l|}{ Pedipalpo } \\
\hline Comprimento total & 16,3 & 17,1 \\
\hline \multicolumn{3}{|l|}{ Tíbia } \\
\hline Comprimento & 4,6 & 5,0 \\
\hline Largura & 1,7 & 1,8 \\
\hline \multicolumn{3}{|l|}{ Fêmur } \\
\hline Comprimento & 3,8 & 4,6 \\
\hline Largura & 1,0 & 1,3 \\
\hline \multicolumn{3}{|l|}{ Mão } \\
\hline Comprimento & 7,9 & 8,1 \\
\hline Largura & 2,2 & 2,3 \\
\hline Altura & 2,3 & 2,3 \\
\hline \multicolumn{3}{|l|}{ Dedo móvel } \\
\hline Comprimento & 4,9 & 5,3 \\
\hline
\end{tabular}

http://www.biotaneotropica.org.br 


\begin{tabular}{|c|c|c|c|c|c|c|}
\hline Espécies & Cristas do metassoma & Dente subaculear & Dentes pectínios & Grânulos no dedo móvel & Comprimento (mm) & Localidade \\
\hline R. acromelas & $10-10-10-8-5$ & $\operatorname{sim}$ & $17-25$ & 8 & $60-65$ & BA,CE,GO,PE,PI \\
\hline R. agamemnon & $10-10-10-8-5$ & $\operatorname{sim}$ & $19-25$ & 8 & $100-110$ & $\mathrm{BA}, \mathrm{CE}, \mathrm{GO}, \mathrm{MT}, \mathrm{PI}$ \\
\hline R. amazonicus & $10-10-10-8-5$ & $\operatorname{sim}$ & 21 & $8-9$ & $50-55$ & $\begin{array}{l}\text { Alter do Châo, } \\
\text { Santarém, PA }\end{array}$ \\
\hline R. crassicauda & $10-10-10-8-5$ & vestigial & $20-25$ & $8-9$ & $40-45$ & Roraima \\
\hline R. debilis & $10-10-10-8-5$ & ausente & $15-16$ & 8 & $23-30$ & CE,PB,BA \\
\hline R. guanambiensis & $10-10-8-8-5$ & $\operatorname{sim}$ & 27 & 7 & $30-44$ & Guanambi, BA \\
\hline R. lacrau & $10-8-8-8-5$ & $\operatorname{sim}$ & $16-17$ & 7 & $35-40$ & Itaeté, BA \\
\hline R. piceus & $10-10-10-8-5$ & vestigial & $22-24$ & 10 & $50-60$ & Roraima \\
\hline R. rochai & $10-10-10-8-5$ & ausente & $21-27$ & 8 & $60-72$ & $\begin{array}{c}\text { BA,CE,PB,PE,PI } \\
\text { RN }\end{array}$ \\
\hline
\end{tabular}

Tabela II:Diagnose das espécies do gênero Rhopalurus que ocorrem no Brasil.

restante de sua extensão; carenas oculares laterais, mediais centrais, laterais posteriores com grânulos moderados; sulco medial longitudinal com profundidade acentuada; olhos medianos anteriores ao centro separados por um diâmetro ocular, 3 pares de olhos laterais. Pedipalpo (Figs. 4-6): fêmur pentacarenado; tíbia com sete carenas; mão mais larga que a tíbia e com nove carenas, delicadas a vestigiais, dedos com sete séries de grânulos e presença de grânulos acessórios internos e externos. Pernas com numerosas cerdas ventrais curtas. Tricobótriotaxia: ortobotriotaxia A-? (Vachon, 1974, 1975). Mesossoma: tergitos com granulação moderada, presença de uma carena mediana robusta na metade posterior do tergitos I-VI, grânulos proeminentes e projetados na margem posterior de cada tergito, sendo três mais acentuados, um mediano e outros dois laterais; tergito VII pentacarenado com grânulos moderados, carena medial ocupando a metade anterior e carenas laterais ocupando dois terços anteriores com grânulos robustos, sendo dois apicais maiores. Ventre: esternito III com região mediana triangular lisa e elevada, duas áreas granulosas ovaladas sob os pentes formando o aparelho estridulatório; II-III planos medialmente com granulação delicada ao redor dos estigmas; IV com granulação esparsa e finamente pontuada medialmente, acentuando-se ao redor dos estigmas; V com quatro carenas com grânulos moderados, carenas laterais internas posicionados na metade posterior e carenas laterais externas ocupando dois terços anteriores a partir da margem, estigmas com comprimento três vezes maior que a largura; pentes com 27-27 dentes pectíneos (Fig. 2). Metassoma: segmentos I-II com grânulos espiniformes apicais nas carenas dorsais e dorso-laterais; I segmento com 10 carenas completas e granulação robusta; II com oito carenas completas e uma carena lateral de cada lado, ambos com granulação robusta; III com oito cristas completas e dois grânulos laterais proeminentes na margem posterior, carenas dorsais e dorso-laterais acentuadas; IV com oito carenas completas, carenas dorsais e dorso-laterais acentuadas, sulco dorsal profundo; $\mathrm{V}$ com cinco carenas, sendo, três carenas ventrais vestigiais e formação de carenas dorsais com granulações delicadas, incompletas na metade posterior, sulco dorsal moderado; IV-V fortemente dilatados. Telson com vesícula lisa, menor que a largura do I segmento, plana e sem granulação aparente, aguilhão curvo com presença de tubérculo subaculear acentuado, duas projeções dorsais proeminentes próximos à margem anterior. Hemiespermatóforo apresentado nas figuras 9-10.

Fêmea (Parátipo): encontra-se em mau estado de conservação. O colorido e os principais caracteres morfológicos são os mesmos apresentados pelo macho, diferindo apenas pela mão mais estreita e pelos dois últimos segmentos do metassoma não dilatados.

Distribuição geográfica: só registrada da localidadetipo, Guanambi, Estado da Bahia, Brasil. Esta cidade localizase entre os patamares ocidentais da Serra do Espinhaço e patamares da Serra Geral no Vale do Iuiú, com presença de campos de vegetação aberta e uma área de transição para uma região de floresta estacional decídua, na Bacia Hidrográfica do Rio São Francisco. 


\section{Agradecimentos}

A todas pessoas que contribuíram para realização deste trabalho (Instituto Butantan, Laboratório de Artrópodes) Denise M. Candido, Antonio D. Brescovit, Cristina A. Rheims, Rafael P. Indicatti, Celina Y. Fukami e Cláudio A. R. de Souza, Dr. Wilson R. Lourenço (Muséum National d'Histoire Naturelle, Paris, França) e a FAPESP projeto $n^{\circ} 02 / 14027-3$.

\section{Referências Bibliográficas}

ACOSTA, L.E. \& MAURY, E.A. 1990. Estridulacion en Timogenes elegans (Mello-Leitão) (Scorpiones, Bothriuridae). Boln Soc. Biol. Concepción 61: 29-37.

FET, V., SISSOM, W.D., G. LOWE \& BRAUNWALDER, M. E.. 2000. Catalog of the Scorpions of the World (1758-1998). The New York Entomological Society, New York, 690 pp.

LOURENÇO, W.R. 1979. A propôs de la véritable identité des genres Rhopalurus Thorell, 1876 et Centruroides Marx, 1889 (Scorpiones, Buthidae). Rev. Arachnol. 2(5): 213-219.

LOURENÇO, W.R. 1982. Révision de genre Rhopalurus Thorell, 1876 (Scorpiones, Buthidae). Rev. Arachnol., 4: 107-141.

LOURENÇO, W.R. 1986. Biogéographie et phylogenie des scoprions du genre Rhopalurus (Scorpiones, Buthidae). Mém. Soc. R. Belge Ent., 33: 129-137.

LOURENÇO, 2002. Scorpions of Brazil. Les Éditions de l'If. 307 p.

LOURENÇO W.R. \& CLOUDSLEY-THOMPSON J.L. 1995. Stridulatory apparatus and the evolutionary significance of sound production in Rhopalurus species (Scorpiones: Buthidae). J. Arid. Envir. 31(4): 423-429.

MELLO-LEITÃO, C. de. 1945. Escorpiões Sul-Americanos. Arquivos do Museu Nacional 40: 1- 468.

POCOCK, R. I. 1904. I. The Collections of William John Burchell, D. C. L., in the Hope Department, Oxford University Museum, II. On a new Stridulating-organ in Scorpions discovered by W. J. Burchell in Brazil in 1828. Ann. Mag. Nat. Hist., 13(7): 56-62.

VACHON, M. 1974. Études des caractères utilisés pour classer les familles et les genres de Scorpions (Arachides). 1. La trichobothriotaxie em Arachnologie. Singles trichobothriaux et types de trichobothriotaxie chez les Scorpions. Bull. Mus. Nat. Hist. Nat. $3^{\circ}$ série, $n^{\circ}$ 140, Zoologie 104: 857-958.

VACHON, M. 1975. Sur l'utilisation de la trichobotthriotaxie du bras des pédipalpes des Scorpions (Arachnides) dans le classement des genres de la famille des Buthidae Simon. Comptes rendus des Séances de l'Académie des Sciences 281(D): 1597-1599.
Título: Descrição de uma nova espécie de Rhopalurus Thorell, 1876 (Scorpiones, Buthidae) do nordeste brasileiro.

Autores: Ângelo Ricardo I. P. Lenarducci, Ricardo Pintoda-Rocha \& Sylvia Marlene Lucas

Biota Neotropica, Vol. 5 ( número 1a): 2005

http://www.biotaneotropica.org.br/v5n $1 \mathrm{a} / \mathrm{pt} /$ abstract?taxonomic-review+bn017051a2005

Recebido em 07/12/2003 - Publicado em 01/02/2005

ISSN 1676-0603 